\title{
Motion of Charged Micro-particle Immersed in Liquid Crystal Controlled by In-plane Field for Electro Paper Display
}

\author{
In-Su Baik, Ju-Hwan Choi, Byoung Sun Jung, Sang Youn Jeon, \\ Eun Kyoung Song, and Seung Hee Lee ${ }^{\mathrm{a}}$ \\ School of Advanced Materials Engineering, Chonbuk National University, \\ 664-14 Duckjin-dong, Duckjin-gu, Chonju-si, Chonbuk 561-756, Korea \\ ${ }^{\mathrm{a} E}$-mail : $\underline{\text { 1sh1@chonbuk.ac.kr }}$
}

(Received December 2 2005, Accepted January 2 2006)

\begin{abstract}
We have studied the motion of charged micro-particles that are immersed in a nematic liquid crystal (LC) and controlled by in-plane field. The LC is an anisotropic liquid such that the viscosity of the LC depends on flow direction, phase of the LC, and temperature, which affects the motion of the charged particles under the influence of electric field. This study shows that the motion of charged particles mainly depends on the applied voltage and the LC phase, but does not show any significant influence from the initial alignment of LC, although one may expect directional difference in drag force due to interaction between LC and particle. The viscosity changes due to temperature variations in nematic phase also show no signification influence on particle velocity when compared to the effect from varying in-plane field strength.
\end{abstract}

Keywords : Micro-particle, Electro paper display, Liquid crystal

\section{INTRODUCTION}

New concepts for the information delivery free from time and space limitation are being pursued lately. In realizing such a concept, a display is of important as a bridge between human beings and information. Electronic paper displays (EPD) optimizing merits of plain paper and display device are expected to fulfill that purpose in the near future. Recently, many studies have been conducted to embody electronic paper display. Gyricon[1], e-ink[2], microcup[3], mobile fine particle display (MFPD) [4], in-plane electro-phoretic display[5], electro-wetting display[6] methods were outcome of that effort. In particular, the EPD with electrophoretic method utilizes dispersed particles in the organic solvent. In an electrophoretic EPD, on/off status was decided by controlling movement of the charged particles, which is driven by applied dc voltage. Therefore, response time of the system has direct relation to the speed of particle which is affected by magnitude of applied voltage and viscosity of solvent related to the drag force. In the MFPD, nematic liquid crystal (NLC) was utilized as a solvent. The NLC has an anisotropic shape so that its flow viscosity depends on flow direction. Furthermore, it reorients responding to the applied electric field due to the dielectric anisotropy, causing hydrodynamic flow by NLC reorientation. However, previous work did not make any comments on an initial LC alignment and flow effects on charged particle.

In this study, the movement of the charged particles dispersed inside liquid crystal was controlled by in-plain electric field. To understand orientation and flow effects of the NLC on motion of the charged particles, two homogeneous aligned LC cells that has initial orientation of parallel and nearly perpendicular to the in-plane field were fabricated. Furthermore, particle's reactions to the in-plain field were carefully examined with different conditions such as the temperature, the LC phase, and the amplitude of applied voltage.

\section{EXPERIMENTS}

The basic cell structure used in the present experiment is depicted in Fig. 1. The bottom substrate has interdigitized electrodes made of aluminum with a distance of $30 \mu \mathrm{m}$ between electrodes and electrode width of $10 \mu \mathrm{m}$, where each electrode does a role of signal and counter electrode. The top substrate do not have electrode, so that with applying voltage, the in-plane field is mainly generated. The homogeneous alignment layer (AL16139 from Japan Synthetic Rubber) was coated on both substrates with thickness of $800 \AA$. The super fluorinated NLC mixture from Merck-Japan (dielectric anisotropy 


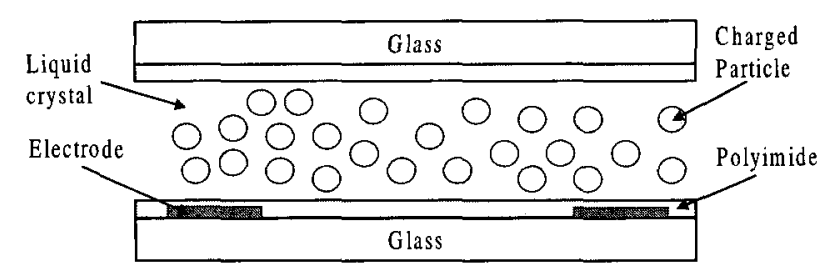

Fig. 1. Schematic cell structure of the device used in this experiment.

$(\Delta \varepsilon)=8.1$ at $1 \mathrm{kHz}$, clearing temperature $\left(\mathrm{T}_{\mathrm{c}}\right)=72^{\circ} \mathrm{C}$, flow viscosity $=78 \mathrm{~mm}^{2} \mathrm{~s}^{-1}$ at $20^{\circ} \mathrm{C}$ ) was used. White particles of $5 \mu \mathrm{m}$ diameter positively charged (Sekisui Fine Chemical, SP-205) were dispersed in the LC with the ratio of $0.2 \mathrm{wt} \%$. The mixture was then filled into the cell by dropping method. Then, both substrates were assembled with a cell gap (d) of $60 \mu \mathrm{m}$. Two samples having initial NLC orientation angles of $0^{\circ}$ and $80^{\circ}$ with respect to in-plane field direction were fabricated. The motion of particles was observed under the optical polarizing microscopy (Nikon E600POL with DXM 1200 digital camera) by applying dc voltages.

\section{BASIC THEORY OF CHARGED PARTICLES UNDER EXTERNAL FIELD}

Micrographs and illustration of particles' motion shown in Fig. 2 explains how the particle reacts to the applied dc voltage. Figure 2(a) shows a charged particle between electrodes in a zero-voltage state. When the dc voltages are applied, the charged particle moves back and forth between electrodes following the changes in the field direction, as shown in Fig. 2(b) and 2(c). During the movements of particle, it experiences resistance of $\mathrm{LC}$ generating drag force, since the NLC is viscous fluid.

Since the LC used in this experiment has positive dielectric constant, it tries to orient parallel to the electric field. In case of cell with $0^{\circ}$ rubbing, the LC does not experience twist deformation at all since the director is parallel to the in-plane field. Therefore, rotation of LC does not take part in the movement of the particles under the influence of the field. On the contrary, for a cell with $80^{\circ}$ alignment layer, the LC experiences mainly twist deformation along the field direction and with enough high voltage, rotation of LC toward the direction of induced field occurs when the direction of the electric field is different from the rubbing direction since the LC aligns in rubbing direction initially. In that case, the movement of particle is affected by the rotation of LC under the influence the field. The $\mathrm{LC}$ reacts to the applied field much faster than the particle. Therefore, reaction of LC by the applied voltage may induce some interaction to the particle before it reacts to the field. Hence, the difference in LC rotation due to the alignment of LC should affect the change in speed of particles. To investigate this effect, sample angle between rubbing and in-plane field direction $\left(80^{\circ}\right)$ was chosen and compared with the case when the rubbing direction is parallel to field direction $\left(0^{\circ}\right)$.

The movements of charged particles may be affected by the elements such as field strength, particle size, viscosity of LC, temperature, LC modes, and cell gap d. In our experiments, effects from these elements were carefully analyzed. Therefore, understanding the effects from these elements is absolutely necessary and we will
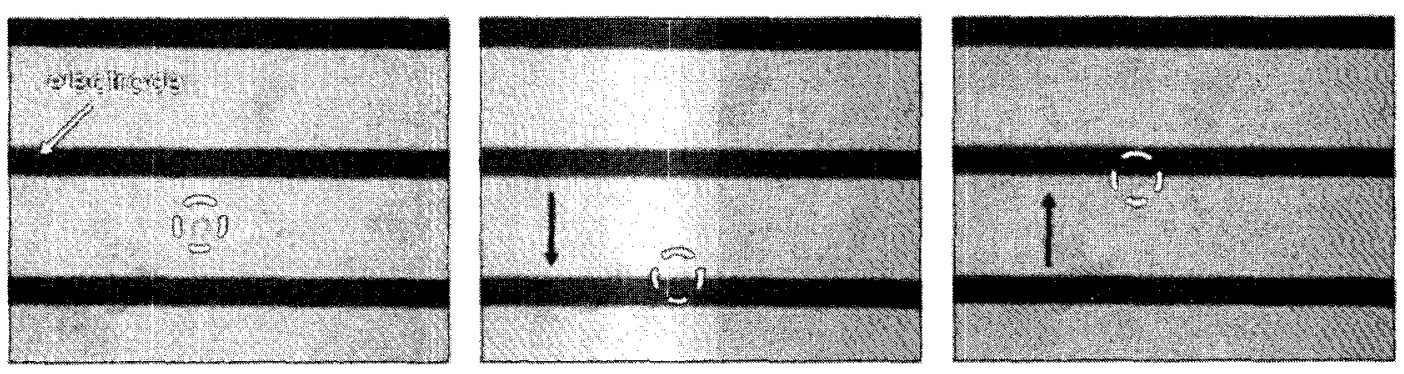

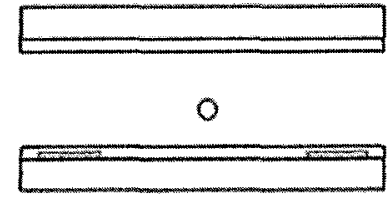

(a)

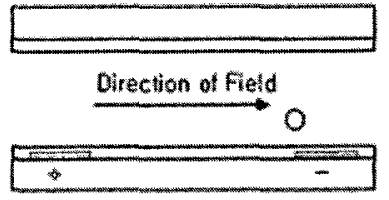

(b)

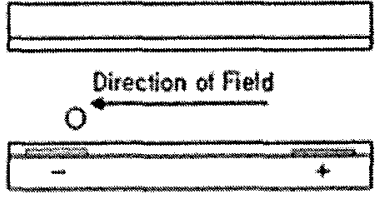

(c)

Fig. 2. Illustration and micrographs show directions of the moving particles due to the applied DC voltage, (a) field off state, (b) + DC applied state, (c) when - DC applied state. 


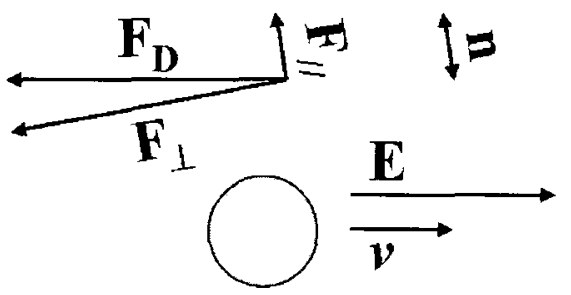

Fig. 3. Illustration shows directions of drag force $\left(\mathbf{F}_{\mathfrak{D}}\right)$, LC director (n), particle movement (v), and electric field (E).

discuss these features below. First, the motions of particles are directly related to the strength of field that affects on particles. The changes in field strength by increasing the applied voltage increased the particle's speed. Second, viscosity of the LC is known to dependent on temperature. It is well-known that, with temperature increment of $10{ }^{\circ} \mathrm{C}$, viscosity drops to one half and this relation shows that variation of the viscosity by changing the temperature have effect on particle speed. In the present work, the temperature was also varied to find the effect of viscosity on the movement of particles. At last, the movement of particles were measured when LC phase are nematic and isotropic in order to find the effect of the LC phase change.

Now, the basic mechanics and relations between the motion of particles and external conditions are explained. The motion of particles dispersed in LC by an external force $\boldsymbol{F}$ is governed by

$$
m a=F-b v
$$

where $\boldsymbol{a}$ and $\boldsymbol{v}$ are the acceleration and velocity of the particles and viscosity $\eta$ generates drag force term (b v). The friction drag term due to viscosity $\eta$ can be determined by the well known Stokes' formula $\boldsymbol{F}_{\mathrm{D}}=-6$ $\pi \mathrm{r} \eta v$, which show the relation between the viscosity drag force, the radius $r$ of the particle and its velocity $v$. From this equation, the effects of drag force which arose from viscosity can be explained. The relationship between temperature and viscosity can also be considered subsequently in order to understand the motion of particles. Also, the effect of rubbing takes part in the reaction of particles to the applied field because the drag force is related to the LC director field. Figure 3 depicted the drag force, LC director field, direction of in-plane field, and direction of charged particle.

In nematic liquid crystals, the drag force also depends on the geometry of the system, i.e., anisotropy of drag force in the system. Using a liquid crystal of rod-like molecules, it is easier to move the particle parallel to the general director field (n), i.e., along the long axis of the molecules, than to move it perpendicular to the director, i.e., perpendicular to the long axis, therefore the director field configuration may have impact on the particle movement. The introduction alignment by rubbing made the LC director field (n) to change. The change of LC alignment or variation in director field induces changes in disclination loop around particles which will have effect on particle movement.

The differences in anchoring of LC to particles may affect the movement of particle, as reported previously $[7,8]$. In the case of high anchoring energy, the drag force to the particles are larger than that of the low anchoring energy case because with low anchoring energy the director field is less disturbed by anchoring of LC to the particles and show almost uniform director field distribution. Ruhwandl and Terentjev reported that the drag force $\mathrm{F}_{\mathrm{D}}$ is reduced in uniform director field and drag force in the parallel direction $\mathrm{F}_{/ /}$is less than that of the isotropic case[9]. In their report, the anisotropy of drag force is increased to 1.69 when the director field is uniform. When particles are charged, the anchoring of LC to the particle may become stronger and therefore the drag force becomes larger but this strong boundary condition results less anisotropy in viscosity. Hence, the difference between drag force parallel $\left(F_{/ /}\right)$and perpendicular $\left(F_{\perp}\right)$ to director field becomes smaller. Therefore, the director field configuration effect is less dominant in drag force in case of charged particles even though the drag force itself was increased.

\section{RESULTS AND DISCUSSION}

Particle movements were measured with different $d c$ applied voltages for samples with rubbing angle of $0^{\circ}$ and $80^{\circ}$ with perpendicular to electrode as well as incase of different temperatures and LC modes. Figure 4 shows the changes in velocity of charged particles as a function of applied voltages for the samples with two different rubbing angles. With increments of applied dc voltage from 7 to $10 \mathrm{~V}$, the speed of charged particle increased from 38.7 to $96.9 \mu \mathrm{m} / \mathrm{s}$ in proportion to the strength of the voltage in case of $0^{\circ}$ rubbing sample. Changing the rubbing angle should affect the speeds of the particles, simply because the particles undergo different LC director field configurations. Nevertheless, the changes in velocity of particles due from changing the rubbing angle were not noticeable (within error range). At $7 \mathrm{~V}$, the average speed of particles was 36.7 $\mu \mathrm{m} / \mathrm{s}$ for $80^{\circ}$ rubbing and $38.7 \mu \mathrm{m} / \mathrm{s}$ for $0^{\circ}$ rubbing with error range of about $6 \sim 7 \mu \mathrm{m} / \mathrm{s}$. With increased applied voltage at $10 \mathrm{~V}$, the average speed of particles became $92.7 \mu \mathrm{m} / \mathrm{s}$ with $80^{\circ}$ rubbing and $96.9 \mu \mathrm{m} / \mathrm{s}$ with $0^{\circ}$ rubbing with error range of about 18 and $10 \mu \mathrm{m} / \mathrm{s}$, 


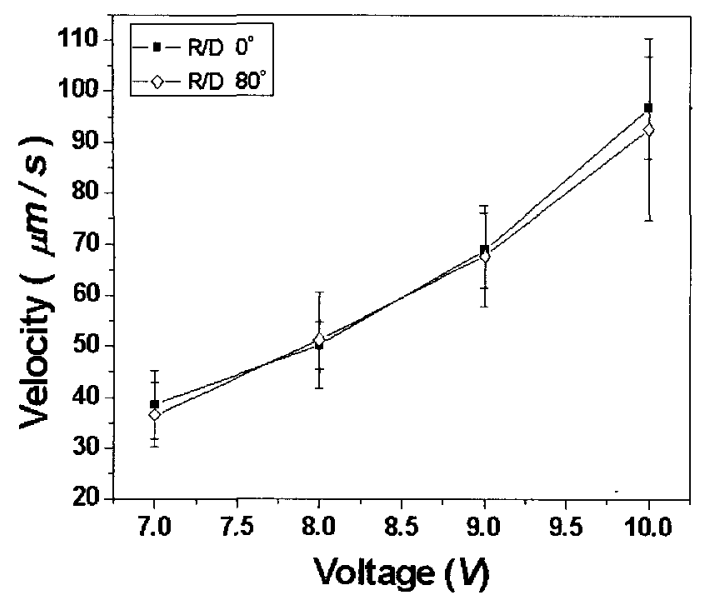

Fig. 4. Effect of the various applied voltage on charged micro-particles when rubbing directions are $0^{\circ}$ and $80^{\circ}$, respectively.

respectively. This suggests the rubbing direction does not affect much on the speed of the particle. The change of speed due to LC alignment by rubbing is within the error margin even though the average speed is decreased a small bit.

Previously, the anchoring of LC to micro-sized particle was studied[9]. This anchoring of LC may affect the movement of the charged particle when field is applied. The interaction between LC and particle i.e., anchoring of LC at the surface of particle results disturbance in the LC alignment or director field. This disturbance may affect the speed of particle when voltage is applied. But, differences in LC director field did not affect the particle movement much as shown in Fig. 4. This proved that the change in anchoring condition due to different $\mathrm{LC}$ director field and the effect of LC orientation to the particle movement is much less dominant feature at particle speed when dc voltage was applied.

As stated earlier, in rode-like molecule LC, particles move much easier in the director field direction. Hence, rotation of LC should affect the particle movement due to changes in director field as well as directional differences in drag force. With applied voltage of $10 \mathrm{~V}$, only LC near substrate is affected by electric field and therefore rotation of LC did not occur at whole region since the cell gap d used at the present experiment is 60 $\mu \mathrm{m}$ which is large compared to the size of particle. This may also add to the reason why the little difference in particle movement when the rubbing direction was varied.

Regions of high gradients (strong anchoring generates large disturbance in LC orientation specifically around the particle) of director field results in higher resistance to particle movements. This is mainly due to boundary condition on the particle from high anchoring energy

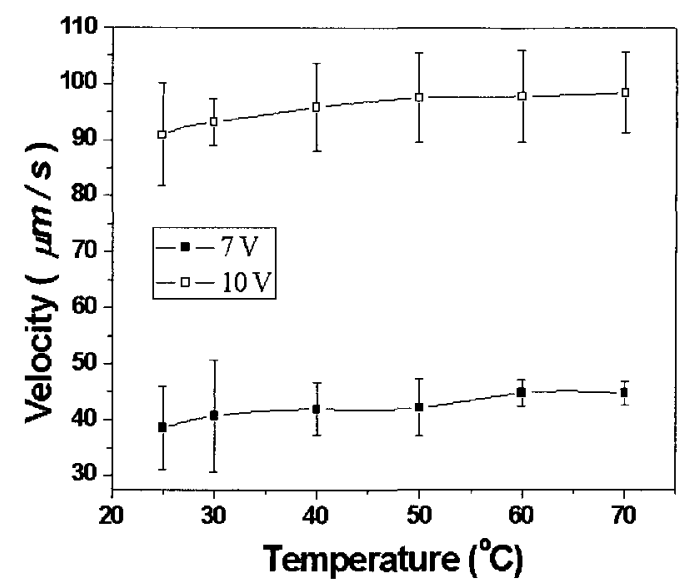

Fig. 5. Variation of temperature affects the velocity of particles when applied voltages were $7 \mathrm{~V}$ and $10 \mathrm{~V}$, respectively.

which is directly related to drag force. This means that stronger boundary condition lowers the anisotropy of director field as well as effects from the drag force anisotropy. From previous report, the charged particle have strong anchoring and exhibit lower anisotropy of drag force than particle in uniform director field.

Figure 5 shows the particle speeds which were measured with variation of temperature for applied voltage of $7 \mathrm{~V}$ and $10 \mathrm{~V}$. The speed measurement of particles did not show any significant increase for both cases even with the increment of the temperature. At $25^{\circ} \mathrm{C}$, the average speed of particles was $38.5 \mu \mathrm{m} / \mathrm{s}$ at 7 $\mathrm{V}$ and $90.9 \mu \mathrm{m} / \mathrm{s}$ at $10 \mathrm{~V}$ with error range of about $6 \sim 7$ $\mu \mathrm{m} / \mathrm{s}$. At $70^{\circ} \mathrm{C}$, the average speed of particles was 44.7 $\mu \mathrm{m} / \mathrm{s}$ at $7 \mathrm{~V}$ and $98.4 \mu \mathrm{m} / \mathrm{s}$ at $10 \mathrm{~V}$ with very small error margin. The increase of average speed is $7.45 \%$ at $7 \mathrm{~V}$ and $3.96 \%$ at $10 \mathrm{~V}$ but the viscosity decreased much more with increased temperature at the same time. This suggests that the movement of particles was not much affected by the change of viscosity when applied voltage is large enough to allow particles to move.

At first, we expected that the variation of temperature affects the speed of particles because the viscosity of the LC changes with temperature variation. The drag force is reduced when the temperature is increased and also the drag force $\left(F_{\perp}\right)$ perpendicular to the LC director is may significantly decreased with increment of temperature. Nevertheless, the drag force $\left(F_{/ /}\right)$parallel to the LC director shows small changes to a lower value at the transition point and stays almost constant independent of temperature when the temperature was lowered[9]. Since the particles only experience the drag force $\left(F_{/ /}\right)$parallel to the LC director, changes in speed of particles due to drag force become relatively small when the electric field is parallel while the temperature is changed. This 


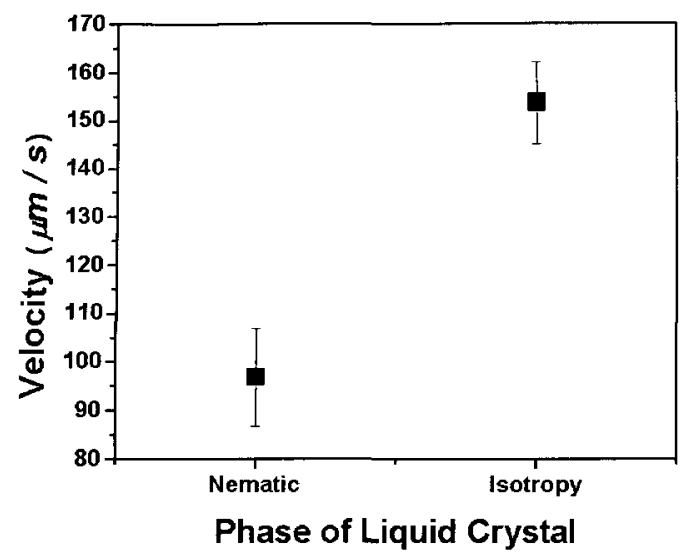

Fig. 6. Effect of liquid crystal phase on velocity of particles when applied voltage is $10 \mathrm{~V}$.

is the reason why the temperature increment does not boost the particle speed much.

The changes in the particle movement due to the difference in LC phase were one of our interests. Figure 6 shows measurements of particle speed when LC phase were nematic and isotropic. As mentioned earlier, the speed of particles does not change much due from the increase of temperature, i.e. viscosity drop. Above the $T_{c}$, the phase of LC changes to isotropic and the speed of particles suddenly increase. This change in particle speed is originated from sudden change in viscosity and drag force $F_{D}$. In case of nematic state, the speed of particles was measured at $25^{\circ} \mathrm{C}$ with average values of $96.9 \pm$ $10.13 \mu \mathrm{m} / \mathrm{s}$. Then, temperature was raised to $80{ }^{\circ} \mathrm{C}$ for isotropic sate and particle speed were measured allowing enough time to reach isotropic state and show average value of $153.9 \pm 8.47 \mu \mathrm{m} / \mathrm{s}$. The speed of particle was about $50 \%$ increased when compared with that of $70^{\circ} \mathrm{C}$.

In these experiments to find the effect of LC modes, applied voltages were set to $10 \mathrm{~V}$ for both nematic and isotropic modes. As seen in Fig. 6, it clearly shows that the particle movement is strongly dependent on the phase of LC even for same applied voltage. The particle velocity was significantly increased when LC phase changed to isotropic, i.e., the particles suspended inside nematic LC are less mobile than those inside isotropic LC. Above $\mathrm{T}_{c}$, the viscosity of $\mathrm{LC}$ become isotropic and the drag force parallel $\left(\mathrm{F}_{/ /}\right)$and perpendicular $\left(\mathrm{F}_{\perp}\right)$ to director should be same since the viscosity anisotropy is no longer exist and therefore, the speed of particle drastically increased above $T_{c}$.

\section{CONCLUSION}

We have introduced charged white micro-particles in nematic liquid crystal (NLC) and studied their behaviors under the influence of the electric field by applying voltages which will provide great deal of information for the possible application in the electro-phoretic type electronic paper display (EPD). The motion of microparticles were studied with different LC cell conditions such as, temperatures, LC director field configurations, and LC modes, which are the factors that affect the movement of particles dispersed inside liquid crystal in this experiment. In the nematic phase, the speed of charged particle were increased in proportion to the applied voltage but the differences in LC conditions such as rubbing angle as well as temperatures only show minute impact on particles' movement. The anchoring conditions and anisotropy of drag force were tightly related to these small changes in the speed of particles. Therefore, changes in the speed of particles are mainly related to the increment of applied voltage. However, when the temperatures were increased enough to change the phase of LC, the speed of particles were significant increased due to the decrease in drag force.

In conclusion, the charged white micro-particles were successfully controlled by applying dc voltage in the nematic LC showing that the usefulness of LC as a solvent in electro-phoretic type EPD applications.

\section{ACKNOWLEDGMENTS}

This work was supported by the Korea Research Foundation Grant funded by the Korea Government (MOEHRD) KRF-2005-202-D00233.

\section{REFERENCES}

[1] T. Pham, N. Sheridon, and R. sprague, "ElectroOptical Characteristics of the Gyricon Display", SID 02 Digest, p. 119, 2002.

[2] B. Comiskey, J. D. Albert, H. Yoshizawa, and J. Jacobson, "Electrophoretic ink for all-printed reflective electronic displays", Nature, Vol. 394, p. 253, 1998.

[3] R. C. Liang, J. Hou, J. Chung, X. Wang, C. Pereira, and Y. Chen, "Active and Passive Matrix Electrophoretic Displays by Roll-to-Roll Manufacturing Processes", SID 03 digest, p. 838, 2003.

[4] M. Ogawa, T. Takahashi, S. Saito, Y. Toko, Y. Iwakura, K. Kobayashi, and T. Akahane, "Behavior of Fine Particles in Mobile Fine Particle Display Cells", SID 03 Digest, p. 584, 2003.

[5] E. Kishi, Y. Matsuda, Y. Uno, A. Ogawa, T. Goden, N. Ukigaya, M. Nakanishi, T. Ikeda, H. Matsuda, and K Eguchi, "Development of In-Plane EPD", SID Tech. Digest 31, p. 24, 2000. 
[6] R. A. Hayes and B. J. Feenstra, "Video-speed electronic paper based on electrowetting", Nature, Vol. 425, p. 383, 2003.

[7] O. V. Kuksenok, R. W. Ruhwandl, S. V. Shiyanovskii, and E. M. Terentjev, "Director structure around a colloid particle suspended in a nematic liquid crystal", Phys. Rev. E, Vol. 54, No. 5, p. 5198, 1996.
[8] S. Grollau, N. L. Abbott, and J. J. de Pablo, "Spherical particle immersed in a nematic liquid crystal: Effects of confinement on the director field configurations", Phys. Rev. E, Vol. 67, No. 1, p. 011702, 2003.

[9] R. W. Ruhwandl and E. M. Terentjev, "Friction drag on a particle moving in a nematic liquid crystal", Phys. Rev. E, Vol. 54, No. 5, p. 5204, 1996. 